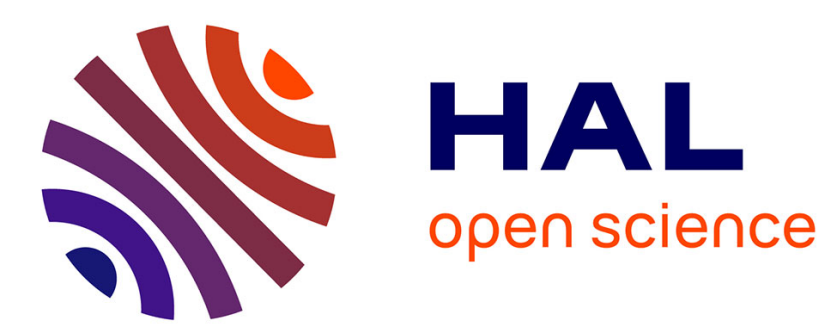

\title{
Cosserat elastoplastic finite elements for masonry structures
}

Michele Godio, Ioannis Stefanou, Karam Sab, Jean Sulem

\section{To cite this version:}

Michele Godio, Ioannis Stefanou, Karam Sab, Jean Sulem. Cosserat elastoplastic finite elements for masonry structures. Mechanics of Masonry Structures Strengthened with Composite Materials, Sep 2014, Ravenna, Italy. pp.131 - 138, 10.4028/www.scientific.net/KEM.624.131 hal-01114447

\section{HAL Id: hal-01114447 \\ https://hal.science/hal-01114447}

Submitted on 9 Feb 2015

HAL is a multi-disciplinary open access archive for the deposit and dissemination of scientific research documents, whether they are published or not. The documents may come from teaching and research institutions in France or abroad, or from public or private research centers.
L'archive ouverte pluridisciplinaire HAL, est destinée au dépôt et à la diffusion de documents scientifiques de niveau recherche, publiés ou non, émanant des établissements d'enseignement et de recherche français ou étrangers, des laboratoires publics ou privés. 


\title{
Cosserat elastoplastic finite elements for masonry structures
}

\author{
Michele Godio ${ }^{1, ~ a ~}{ }^{*}$, loannis Stefanou ${ }^{1, b}$, Karam Sab ${ }^{1, ~ c}$, Jean Sulem ${ }^{1, d}$ \\ ${ }^{1}$ Université Paris-Est, Laboratoire Navier (UMR 8205), CNRS, ENPC, IFSTTAR, France \\ amichele.godio@enpc.fr, bioannis.stefanou@enpc.fr, ckaram.sab@enpc.fr, djean.sulem@enpc.fr
}

\begin{abstract}
Keywords: Masonry · Cosserat dynamics · Multi-surface plasticity · Finite Elements · Discrete Elements
\end{abstract}

\begin{abstract}
A Finite Elements formulation previously developed for Cosserat elastic plates, has been extended herein to the elastoplastic framework. Material non-linearities are taken into account through the implementation of a backward-Euler closest-point-projection algorithm, for which the definition of non-smooth yield loci and non-associated plastic potentials and evolution laws is made possible. An existing homogenized elastic constitutive model and a set of yield criteria for the outof-plane behaviour of block-masonry are implemented in the code and their validity is discussed based on the comparison with Discrete Elements simulations. The comparison is carried out in both the static and the dynamic regime.
\end{abstract}

\section{Introduction}

Seismic loadings are a major risk that causes the collapse of masonry buildings. For this reason, nowadays building Codes require the assessment of the seismic vulnerability of masonry structures, together with the estimation of their residual loadbearing capacity after earthquake occurrence, see Eurocode 8. Under this scope, the development of reliable and sophisticated physical models is an essential task and the use of continuum models based on homogenization methods often results in a good compromise between solution accuracy and computational cost.

The use of Cosserat continuum models is particularly well suited for the description of masonry structures, since it allows high deformation gradients, relative block rotations and scale effects to be efficiently taken into account, which is not permitted by equivalent models based on Cauchy continuum [1]-[3]. Moreover, Cosserat continuum permits the investigation of the phenomenon of wave dispersion, governing the dynamic response of periodic masonry [4], [5].

Within this context, a Cosserat Finite Elements (FE) formulation has been developed, with the aim of carrying out elastoplastic dynamic analyses on masonry structures. This formulation is the extension of a previously one, see [6], developed for elasticity and dynamics, which deals with a small-strain plate element, possessing 6 degrees-of-freedom (DOF) per node and allowing quadratic polynomial interpolation on both the displacement and the rotational fields. This particular element has been formulated in Abaqus as User ELement (UEL), allowing one to control the analysis at each step of a prescribed procedure and to compute the stress and deformation state at every Gauss point. At present, this makes possible the implementation of incremental elastoplastic constitutive models, through the definition of multiple plastic surfaces, non-associated potentials and specific evolution laws.

The purpose of this paper is to show the efficiency of Cosserat homogenization models in representing the dynamic and the elastoplastic behaviour of masonry, compared to more refined approaches which, even though they allow for a more detailed mechanical description, are computationally expensive and make more difficult the investigation of the overall response of the structure. The first part will be dedicated to present the extension of the Cosserat FE [6] to the elastoplastic framework, by implementation of the multi-surface theory into a robust solution algorithm. In the second part, a comparison will be performed on the illustrative example of a masonry wall, between the results produced by the homogenization models implemented in the code and those obtained from the use of a Discrete Elements (DE) model.

It is stressed that, in all parts, our attention will be restrained to the out-of-plane behaviour of masonry. The need of evaluating the structural response to bending and torsion as well as the lateral 
strength of masonry elements, represents a typical issue in building Codes, since it is important for the overall structural stability of masonry structures under seismic actions.

Common matrix notation is adopted. Symbol d $[\cdot]$ denotes an increment between two successive iterations whereas partial differentiation is indicated by $\partial_{\mathrm{x}}[\cdot]$. Time derivative is $\left[{ }^{\bullet}\right]=\partial_{\mathrm{t}}[\cdot]$.

\section{Formulation of an elastoplastic Cosserat continuum FE code}

The 3D Cosserat field's problem is governed by 6 partial differential equations (PDE) of $2^{\text {nd }}$ order, expressed in terms of the three unknown displacements $\boldsymbol{u}$ and rotations $\boldsymbol{\omega}$, both collected in the vector $s$ :

$$
\boldsymbol{s}=\left[\begin{array}{ll}
\boldsymbol{u}^{\mathrm{t}} & \boldsymbol{\omega}^{\mathrm{t}}
\end{array}\right]
$$

In the case of a plate all the derivatives along the out-of-plane direction, namely $x_{3}$, are supposed to vanish and the problem dimension is reduced (Fig. 1). The remaining components of relative strain $\boldsymbol{\gamma}$ and curvature $\boldsymbol{\kappa}$ can be assembled into a total generalized strain vector $\boldsymbol{\varepsilon}$, whereas the conjugatein-energy generalized stress vector, including stresses and couple-stresses, is $\boldsymbol{\sigma}$ :

$$
\boldsymbol{\varepsilon}=\left[\begin{array}{ll}
\boldsymbol{\gamma}^{\mathrm{t}} & \boldsymbol{\kappa}^{\mathrm{t}}
\end{array}\right] \quad \boldsymbol{\sigma}=\left[\begin{array}{ll}
\boldsymbol{\tau}^{\mathrm{t}} & \boldsymbol{\mu}^{\mathrm{t}}
\end{array}\right] .
$$

Following this notation, the Cosserat PDE of motion read, in Cartesian coordinates:

$$
\left[\begin{array}{rr}
\mathrm{L}_{11}^{\mathrm{t}} & -\mathbf{L}_{21}^{\mathrm{t}} \\
-\mathrm{L}_{12}^{\mathrm{t}} & \mathrm{L}_{22}^{\mathrm{t}}
\end{array}\right]\left[\begin{array}{l}
\tau \\
\mu
\end{array}\right]+\left[\begin{array}{l}
f \\
m
\end{array}\right]-\left[\begin{array}{ll}
1 & 0 \\
0 & \mathrm{I}
\end{array}\right]\left[\begin{array}{l}
\ddot{u} \\
\ddot{\omega}
\end{array}\right]=0
$$

where $\boldsymbol{f}$ and $\boldsymbol{m}$ contain respectively the body forces and the body couples, $\mathbf{1}$ incorporates the mass density and $\mathbf{I}$ the rotary inertia, and $\mathbf{L}_{\mathbf{1 1}}, \mathbf{L}_{\mathbf{1 2}}, \mathbf{L}_{\mathbf{2 1}}, \mathbf{L}_{\mathbf{2 2}}$ are the matrix operators giving the definition of the deformation measures:

$$
\left[\begin{array}{l}
\gamma \\
\kappa
\end{array}\right]=\left[\begin{array}{ll}
\mathbf{L}_{11} & \mathbf{L}_{12} \\
\mathbf{L}_{21} & \mathbf{L}_{22}
\end{array}\right]\left[\begin{array}{l}
u \\
\omega
\end{array}\right],
$$

that is

$$
\begin{aligned}
\mathbf{L}_{11} & =\left[\begin{array}{cccccccc}
\partial_{1} & 0 & 0 & \partial_{2} & 0 & 0 & 0 & 0 \\
0 & \partial_{2} & \partial_{1} & 0 & 0 & 0 & 0 & 0 \\
0 & 0 & 0 & 0 & \partial_{1} & 0 & \partial_{2} & 0
\end{array}\right]^{\mathrm{t}} & \mathbf{L}_{21} & =\left[\begin{array}{cccccc}
0 & 0 & 0 & 0 & 0 & 0 \\
0 & 0 & 0 & 0 & 0 & 0 \\
0 & 0 & 0 & 0 & 0 & 0
\end{array}\right]^{\mathrm{t}} \\
\mathbf{L}_{12} & =\left[\begin{array}{cccccccccc}
0 & 0 & 0 & 0 & 0 & 0 & -1 & +1 \\
0 & 0 & 0 & 0 & +1 & -1 & 0 & 0 \\
0 & 0 & -1 & +1 & 0 & 0 & 0 & 0
\end{array}\right]^{\mathrm{t}} & \mathbf{L}_{22} & =\left[\begin{array}{cccccc}
\partial_{1} & 0 & 0 & \partial_{2} & 0 & 0 \\
0 & \partial_{2} & \partial_{1} & 0 & 0 & 0 \\
0 & 0 & 0 & 0 & \partial_{1} & \partial_{2}
\end{array}\right]^{\mathrm{t}}
\end{aligned}
$$

Multi-surface plasticity for Cosserat materials. In the present FE formulation, the account for plastic strains is made with reference to the theory of multi-surface plasticity. Such theory, which was formulated for classical materials in its original version and then extended for the computation of multiple plastic surfaces [7], has been herein implemented in the frame of the Cosserat continuum, see also [8] and [9] respectively.

Under small strains assumption, the total strain vector can be classically split into its elastic and plastic component, via the additive decomposition:

$$
\mathrm{d} \boldsymbol{\varepsilon}=\mathrm{d} \boldsymbol{\varepsilon}^{\mathrm{el}}+\mathrm{d} \boldsymbol{\varepsilon}^{\mathrm{pl}} .
$$

Elastic strains and curvatures are related to the stress and couple stress vectors through the general constitutive law: 


$$
\mathrm{d} \boldsymbol{\sigma}=\mathbf{D}\left[\mathrm{d} \boldsymbol{\varepsilon}-\mathrm{d} \boldsymbol{\varepsilon}^{\mathrm{pl}}\right]
$$

where $\mathbf{D}$ is the matrix of the elastic moduli. In view of the FE formulation, it is also convenient to express the stresses in function of the total strains, as:

$$
\mathrm{d} \boldsymbol{\sigma}=\mathbf{D}^{\mathbf{e p}} \mathrm{d} \boldsymbol{\varepsilon}
$$

where $\mathbf{D}^{\mathbf{e p}}$ is the matrix of the elastoplastic tangent moduli. The elastic domain $\mathbb{E}_{\sigma}$ can be defined as the convex region of the stress space bounded by multiple, $\mathrm{N}$, prescribed surfaces $f_{\beta}(\boldsymbol{\sigma}, \boldsymbol{q})$, representing the activated yield loci, intersecting in a non-smooth manner, i.e. leading to the presence of corners [7]:

$$
\mathbb{E}_{\sigma}=\left\{(\boldsymbol{\sigma}, \boldsymbol{q}) \mid f_{\beta}(\boldsymbol{\sigma}, \boldsymbol{q}) \leq 0, \forall \beta \in[1, \ldots, \mathrm{N}]\right\}
$$

Non-associative plasticity then requires the definition of plastic potentials $g_{\beta}(\boldsymbol{\sigma}, \boldsymbol{q})$ and hardening functions $h_{\beta}(\boldsymbol{\sigma}, \boldsymbol{q})$, through which define the equations of evolution:

$$
\begin{aligned}
\mathrm{d} \boldsymbol{\alpha} & =\sum_{\beta=1}^{\mathrm{N}} \mathrm{d} \lambda^{\beta} \partial_{\mathbf{q}} h_{\beta}(\boldsymbol{\sigma}, \boldsymbol{q}) \\
\mathrm{d} \boldsymbol{\varepsilon}^{p l} & =\sum_{\beta=1}^{\mathrm{N}} \mathrm{d} \lambda^{\beta} \partial_{\boldsymbol{\sigma}} g_{\beta}(\boldsymbol{\sigma}, \boldsymbol{q})
\end{aligned}
$$

where the hardening law gives the increment of the hardening variable $\boldsymbol{q}$ and of its dual $\boldsymbol{\alpha}$, and the flow rule governs the plastic strain increment.

In presence of multiple surfaces, plastic strains will occur not only from the satisfaction of the yield criteria, but also from the condition on the consistency parameters, i.e.:

$$
\mathrm{d} \lambda^{\beta} \geq 0
$$

The consistency parameters play a crucial role in the formulation of this theory, since they give the measure of the plastic strains and allow one to determine the conditions for which the loading response will be elastic or not. Besides, the unloading response is governed by the same constitutive law which controls the elastic loading. In this sense, damage process is not taken into account in the formulation.

Solution algorithm and FE implementation. The theory of multi-surface plasticity has been incorporated into the present Cosserat FE formulation [6], through the adoption of an implicit (backward-Euler) closest-point-projection (CPP) algorithm.

In particular, for the determination of the active surfaces we employ the Procedure 1 discussed by [7]. The layout of this iterative solution scheme is as follows. Displacements and rotations are first predicted by Abaqus for the generic increment $n+1$ and given to the UEL in terms of nodal DOF $\mathrm{d} \boldsymbol{s}_{\mathrm{n}+1}$. An elastic guess $\mathrm{d} \boldsymbol{\sigma}_{\mathrm{n}+1}^{\mathrm{el}}$ is then evaluated as trial solution, computed from the total strain increment given for the step $\mathrm{d} \boldsymbol{\varepsilon}_{\mathrm{n}+1}$, after Eq. (7). If the elastic guess falls inside the elastic domain, then the new stress state is given directly in addition to the previous one. Otherwise, if at least one of the yield criteria is violated, then Procedure 1 consists in solving the CPP algorithm by holding fixed the set of the activated surfaces and checking solution by testing Eq. (11). Criteria for which such condition is not satisfied are dropped from the set of the active surfaces and a new iteration is required.

Once the elastoplastic solution is calculated at every Gauss Point, the local stiffness tangent matrix and the local mass matrix can be assembled and the residual vector computed. Both are returned to Abaqus, which updates the global solution with that for the given increment and, in turn, recalls the UEL for the next step. 


\section{Comparison between continuum FE and DE solution: application to a masonry wall}

DE and FE methods are two of the most widespread employed numerical procedures in computational structural mechanics. Applied to masonry structures and with reference to homogenization models [10], these methods are used mainly to provide a fine and coarse description of the structural behaviour, through the formulation of, respectively, discrete and continuum models. Discrete models are idealizations in which material regions are separate and their interaction is represented explicitly. In continuum models particles or material bodies are smeared into an equivalent medium and their interaction is only invoked by means of a homogenized constitutive law.

The elastoplastic FE formulation presented in this paper allows the implementation of Cosserat continuum models of the type of a plate. It is in our purpose to evaluate the applicability (and demonstrate the advantages) of such homogenization models against the use of more refined representations, herein the use of DE. Regarding masonry structures, such a comparison is of great importance, since some of the requisites demanded by practitioners can be tested in this way. In particular, it is our intent to investigate: the representation of the out-of-plane behaviour of masonry in the static elastoplastic and dynamic regime; the response sensibility to the boundary conditions; the evaluation of the scale ratio for which the applicability of the homogenized continuum reaches its limits with respect to the discrete solution; the solution accuracy and the calculation cost.

For other investigations on the in-plane response of masonry through linear and non-linear Cosserat homogenized models we refer to [11]-[13], among others. Combined DE/FE formulations for the in-plane and out-of-plane behaviour of masonry can be found in [14] and [15], respectively.
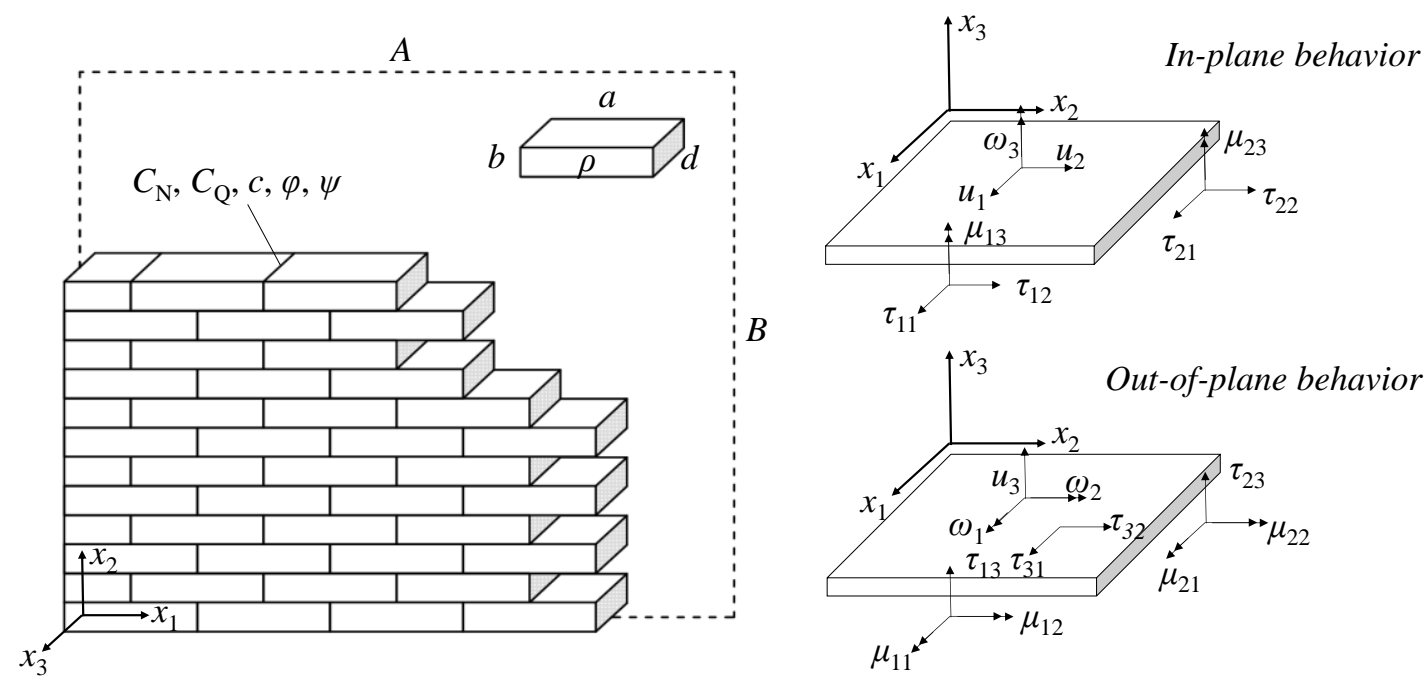

Fig. 1 Statics and kinematics of a Cosserat continuum plate model. Reference to the homogenized running-bond masonry wall studied by [5] and [16].

Continuum FE model. In view of the analysis, we implement the continuum model proposed by [5]. This homogenization model has been derived by identification of a rigid-block running-bond lattice with an equivalent 3D Cosserat elastic continuum, resulting in an orthotropic plate model which is comprehensive of in- and out-of-plane elastic constitutive laws and inertial terms. The parameters entering our formulation are the dimensions of the masonry units $a, b, d$, the normal and shear joint elasticity $C_{\mathrm{N}}, C_{\mathrm{Q}}$ and the mass density of the blocks $\rho$ (Fig. 1).

The elastoplastic behaviour is given thought a set of yield criteria on bending, torsion and transverse shear (Fig. 2). These criteria have been calculated by [16] for a running-bond masonry pattern with Coulomb interfaces, in the framework of limit analysis and homogenization theory. Originally referred to a Reissner-Mindlin plate model, they are implemented in the present code in terms of Cosserat statics. It can be shown from Fig. 1 that the Reissner-Mindlin plate is a special case of the Cosserat plate. Since they are based on the same out-of-plane kinematics, the Cosserat homogenized yield loci can be found, as a first approach, by equating the plastic dissipation energy 
from the two theories. The use of two distinct sets of criteria, the one referred to the pure bending and the other involving only the transverse shear, is justified by [16]. In addition, we formulate a basic criterion for torsion. Plastic surfaces are all expressed in terms of joint cohesion $c$ and friction angle $\varphi$ and are proportional to the vertical stress $\tau_{22}$ (see [16], Eqs.(20)-(21)). Moreover, we define non-associated plastic potentials of the same form of the criteria but with null dilatancy angle $\psi$.

DE model. For the construction of the DE model, we employ the code 3DEC [17]. This program falls within the class of the discontinuous analysis procedures, for which each block, that in our specific case is considered to be rigid, possesses $6 \mathrm{DOF}$ and interact with the adjacent blocks through a number of contact-points detected at interfaces by means of an automatic detection method [18]. The code makes use of an explicit (forward-Euler) time-integration scheme and herein is run in small-strain mode. Plastic deformations are computed by an algorithm similar to that discussed in Section 2.

The actual blocks arrangement and shape are represented in the discrete model as in the lattice studied by [5] and [16]. No calibration of the stiffness parameters is thus necessary and interfaces are governed by the same elastic coefficients $C_{\mathrm{N}}$ and $C_{\mathrm{Q}}$. Moreover, the elastoplastic behaviour is accounted for by considering a perfectly-plastic non-dilatant Coulomb slip criterion at joints. It results that all geometric and material parameters of the discrete model are in agreement with those of the continuum model, which leads to a straightforward comparison between the two proposed modelling approaches.
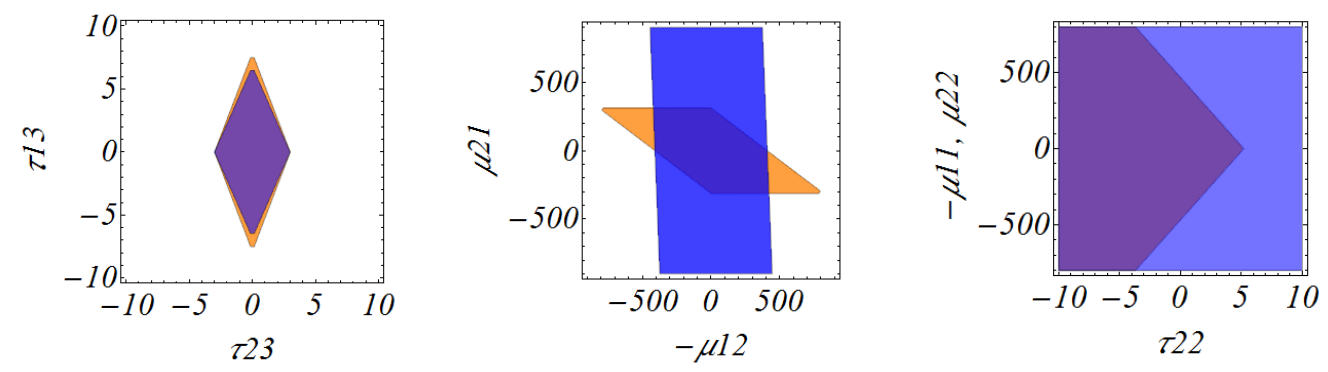

$$
\begin{aligned}
& a=250 \mathrm{~mm} \\
& b=55 \mathrm{~mm} \\
& d=120 \mathrm{~mm} \\
& C_{\mathrm{N}}=80 \mathrm{~N} / \mathrm{mm}^{3} \\
& C_{\mathrm{Q}}=40 \mathrm{~N} / \mathrm{mm}^{3} \\
& c=3 \mathrm{~N} / \mathrm{mm}^{2} \\
& \varphi=30^{\circ} \\
& \psi=0^{\circ} \\
& \rho=20 \mathrm{~kg} / \mathrm{m}^{3}
\end{aligned}
$$

Fig. 2 Homogenized yield loci (light) and plastic potentials (dark) for running-bond masonry, expressed in Cosserat statics. Criteria on transverse shear (left), bending (centre) and torsion (rigth).

Comparison. Two different DE models are examined in order to assess the validity of the continuum FE model (Fig. 3). The first model, which is denoted as Wall $(C)$, consists in a flat unreinforced running-bond masonry wall of uniform thickness and with two adjacent clamped edges, where both block translations and block rotations are prevented. The second model, the Wall $(S)$, has the same geometry as the aforementioned one, but the boundary conditions are somehow different: the wall is maintained on a rigid masonry support on both adjacent sides.

The discrete models are compared to the continuum model, which consists in a Cosserat FE plate with clamped edges. The comparison is carried out on structures with the number of blocks $N_{\mathrm{b}}$ varying simultaneously in both the vertical and horizontal direction (Fig. 3). The thickness $d$ of the wall is kept constant and the wall aspect ratio is fixed as half the block shape factor $m$, i.e. $B / A=$ $b / a=m / 2$. The scale ratio is consequently the inverse of the block number: $b / B=a / A=1 / N_{\mathrm{b}}$. Based on this strategy, modal analyses are made in order to calculate and compare the first natural frequencies and the flexural modal shapes of the discrete and continuum solutions. Moreover, elastic and elastoplastic analyses are carried out with the intent of comparing the response of masonry in the static regime. In this case, a volume load $f_{3}$ is applied by keeping constant the mass density $\rho$ and by increasing the horizontal intensity $G_{3}$. Since the analyses are load-controlled and models have elastoplastic properties, the post-pic (softening) behaviour is not regarded.

The numerical convergence of the continuum FE and DE models against, respectively, mesh and contact-point discretization has been assessed by the study of the first three natural frequencies the walls. According to [19] it is found that a large number of contact-points, 17, are required across the thickness of the joints of the DE model in order to obtain an accurate representation of the bending 
behaviour. This increases remarkably the calculation cost, to FE model's advantage, for which a $8 \times 8$-element subdivision is sufficient.
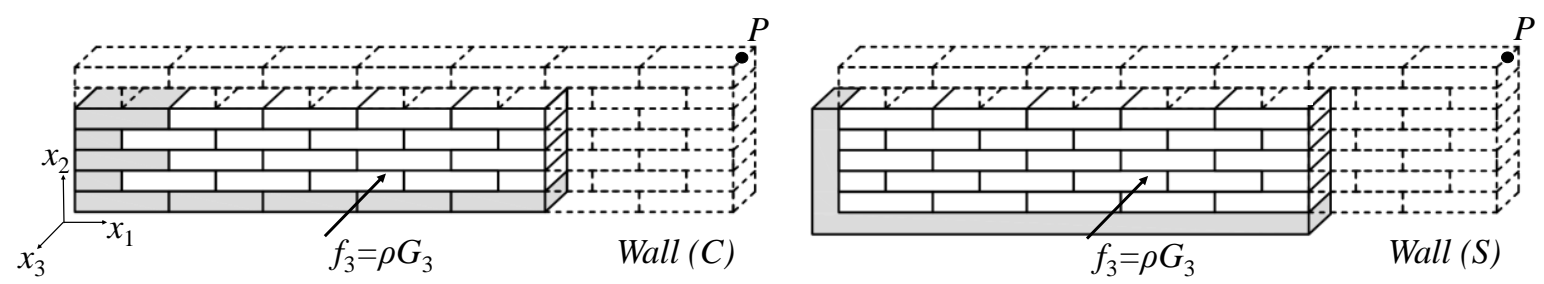

Fig. 3 Sketch of the dicrete models considered for numerical simulations. Left: wall with clamped blocks, Wall $(C)$. Right: wall with rigid block support, Wall $(S)$. Reference to bricks UNI.

In Fig. 4-left we present the comparison in elasticity, by showing the relative error committed by the continuum model in representing the out-of-plane displacement $u_{3}$ at point $P$ of the walls (Fig. $3)$. Results are plotted versus the block number $N_{\mathrm{b}}$, and the relative error is defined as follows:

$$
\epsilon=100 \cdot\left[u_{3}^{\mathrm{DE}}-u_{3}^{\mathrm{FE}}\right] u_{3}{ }^{\mathrm{DE}}{ }^{-1} .
$$

We observe that the FE solution is bounded by the two DE models. In particular, as the block number becomes greater than 25 , the relative error is less than $5 \%$ when compared to the Wall $(S)$, and slightly larger when compared to the Wall $(C)$.
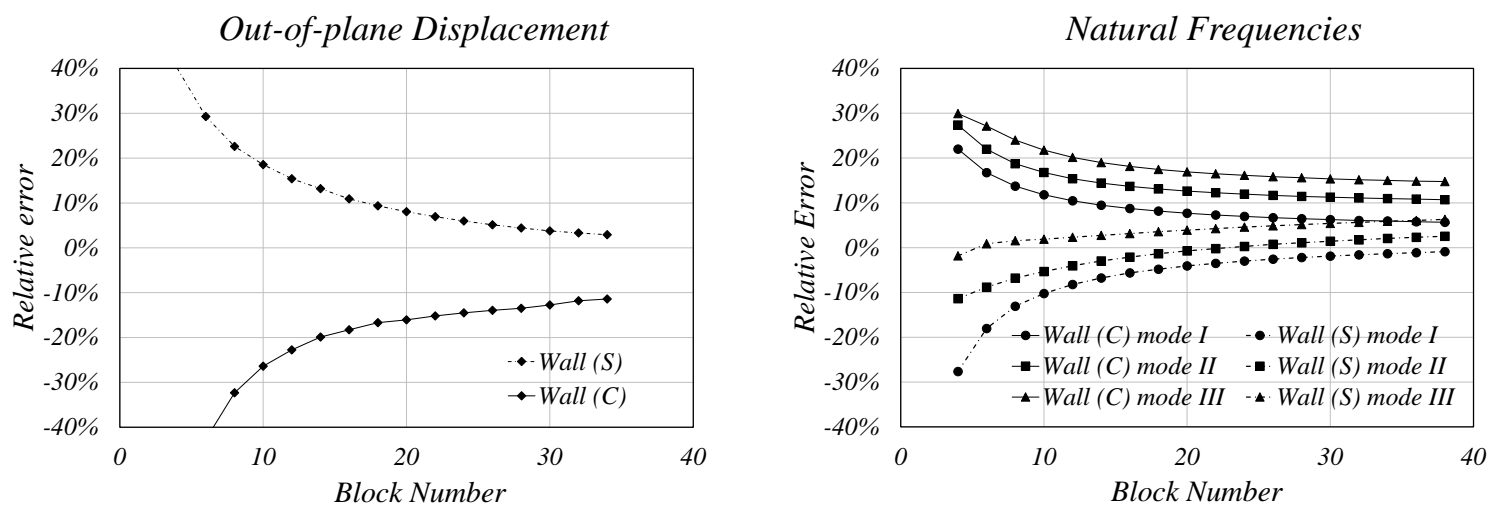

Fig. 4 Relative error between homogenized Cosserat FE and DE models. Left: out-of-plane displacement of point $P$ from static analysis in elasticity. Right: first three flexural frequencies.

In Fig. 4-right we compare the first three natural frequencies of the discrete walls with those given by the continuum model. For the first frequency (mode $I$ ) the relative error is less than 10\% for walls composed with more than 10 blocks. For higher frequencies (mode II and mode III) the error becomes larger, but yet comparable to the precision level assessed in the static analyses. Generally, the increase of the relative error for higher frequency modes is justified by the fact that the homogenized model is a low-frequency approximation of the discrete system [3-4]. Nevertheless, in the present case, the discrepancy in the representation of the higher modes should be rather attributed to the simplified way in which the frequencies are extracted in the DE code [17]. In this code the inertia tensor is considered to be isotropic for each rigid block, and consequently, when torsion is important and blocks are elongated, the computation of the modal frequencies is inexact. However, despite this oversimplification, we observe from the flexural modal shapes of Fig. 5, that the discrete and homogenized solutions are in accordance.

In Fig. 6 the behaviour of the continuum and the discrete models is compared in the elastoplastic regime. The results are given in terms of static normalized load-displacement curves, for walls composed by $8 \times 8,16 \times 16$ and $32 \times 32$ blocks. 
Wall $(C)$
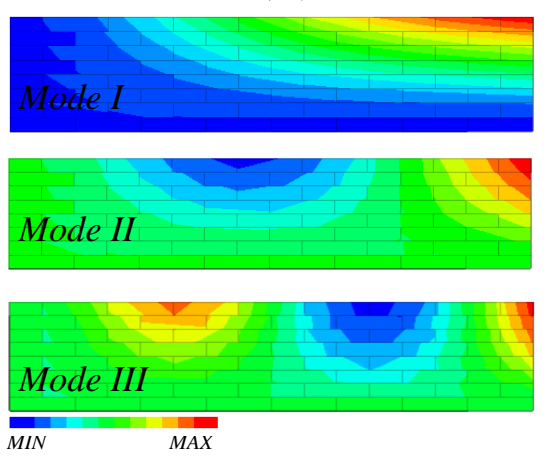

$\operatorname{Wall}(S)$
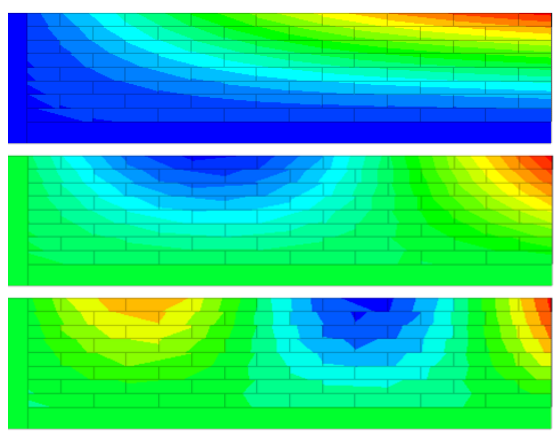

Cosserat FE
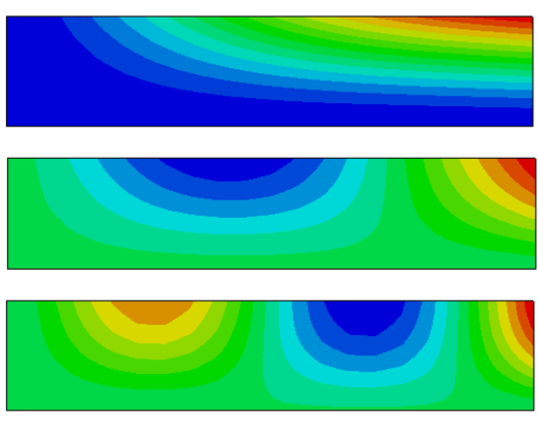

Fig. 5 Out-of-plane-displacement contours of the first three flexural modal shapes. Left and center: DE solution. Right: homogenized Cosserat FE solution. Block number 8.

As expected, the FE solution overestimates the discrete systems, since the simplified expressions for the yield loci used in this example are upper-bound estimates of the actual strength capacity of block-masonry [16]. Nevertheless, the elastoplastic behaviour of the discrete system is quite well captured by the continuum FE representation. It is worth mentioning that a different failure mechanism is observed in both the discrete and the continuum models as the number of the blocks increases. In particular, walls with $8 \times 8$ blocks activate the criterion on transverse shear, which is not the case of more slender 16x16- and 32x32-block walls, where the activation of bending and torsional criteria tends to govern the whole elastoplastic structural response.
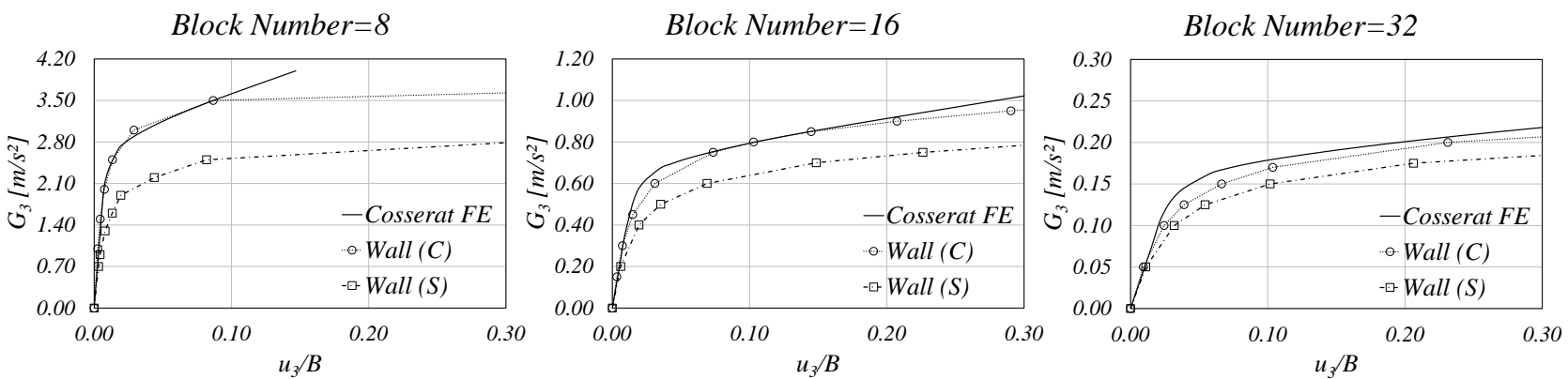

Fig. 6 Comparison between homogenized Cosserat FE and DE solutions in elastoplasticity. Load histories from out-of-plane static analyses for non-associated perfect plasticity.

All the analyses have shown that the regions of the wall in which the stress state of FE model attains one of the yield loci approximately correspond to the zones where inter-block slips are initiated into the DE model (Fig. 7). This makes evident that the failure mechanisms described by the two modelling approaches are, at least qualitatively, in accordance.

\section{Concluding remarks}

The results obtained from the above examples demonstrate the efficiency of homogenized models based on Cosserat continuum to represent the elastic, modal and elastoplastic out-of-plane behavior of block-masonry. It has been shown how such models can be implemented into a suitable FE code, in which the account for irreversible strains can be made by means of a robust quadraticconvergent algorithm. The presented elastoplastic Cosserat Finite Elements formulation constitutes an example, which gives the basis for the creation of a more sophisticated numerical tool to perform the seismic design of masonry structures. In this perspective, the implementation of a complete set of yield loci for the coupled in-plane and out-of-plane behavior of masonry and the introduction of specific evolutions laws for the account of softening and damage processes must be envisaged. 

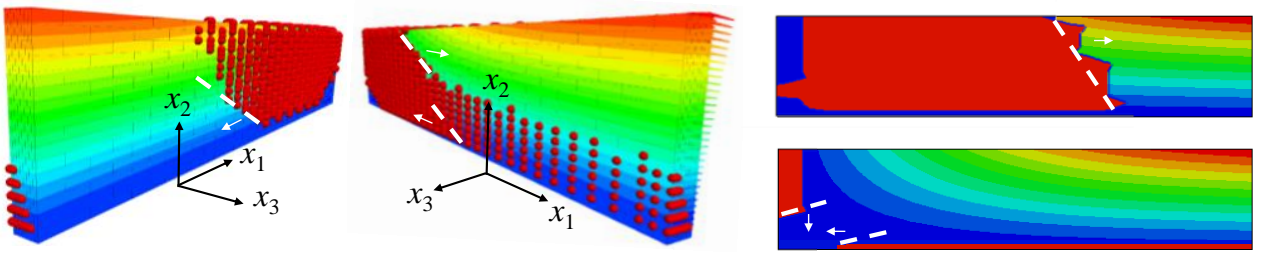

Fig. 7 Left:

developpement of the inter-block slip in the DE model (red dots) by increasing loading. Right: progression of the plastic strains in the homogeneized Cosserat FE model (red zone); activation of criteria on torsion (up) and bending (down). Block Number 16.

\section{References}

[1] G. Salerno and G. de Felice, "Continuum modeling of periodic brickwork," Int. J. Solids Struct., vol. 46, no. 5, pp. 1251-1267, Mar. 2009.

[2] A. Pau and P. Trovalusci, "Block masonry as equivalent micropolar continua: the role of relative rotations," Acta Mech., vol. 223, no. 7, pp. 1455-1471, May 2012.

[3] P. Trovalusci and A. Pau, "Derivation of microstructured continua from lattice systems via principle of virtual works: the case of masonry-like materials as micropolar, second gradient and classical continua," Acta Mech., vol. 225, no. 1, pp. 157-177, Aug. 2013.

[4] J. Sulem and H. Mühlhaus, "A continuum model for periodic two-dimensional block structures," Mech. Cohesive Frict. Mater., vol. 2, no. March 1996, pp. 31-46, 1997.

[5] I. Stefanou, J. Sulem, and I. Vardoulakis, "Three-dimensional Cosserat homogenization of masonry structures: elasticity," Acta Geotech., vol. 3, no. 1, pp. 71-83, Feb. 2008.

[6] M. Godio, "Etude théorique et numérique du comportement dynamique tridimensionnel des structures en maçonnerie," MSc Thesis ENPC, 2012.

[7] J. Simo, J. G. Kennedy, and S. Gonvindjee, "Non-smooth multisurface plasticity and viscoplasticity. Loading/unloading conditions and numerical algorithms," Int. J. Numer. Methods Eng., vol. 26, no. June 1987, pp. 2161-2185, 1988.

[8] R. de Borst, "Simulation of strain localization: a reappraisal of the Cosserat continuum," Eng. Comput., vol. 8, no. 4, pp. 317-332, 1991.

[9] D. Addessi, "A 2D Cosserat finite element based on a damage-plastic model for brittle materials," Comput. Struct., vol. 135, pp. 20-31, Apr. 2014.

[10] P. Trovalusci and R. Masiani, "Material symmetries of micropolar continua equivalent to lattices," Int. J. Solids Struct., 1999.

[11] P. Trovalusci and R. Masiani, "Non-linear micropolar and classical continua for anisotropic discontinuous materials," Int. J. Solids Struct., vol. 40, no. 5, pp. 1281-1297, Mar. 2003.

[12] D. Addessi, E. Sacco, and A. Paolone, "Cosserat model for periodic masonry deduced by nonlinear homogenization," Eur. J. Mech. - A/Solids, vol. 29, no. 4, pp. 724-737, Jul. 2010.

[13] D. Addessi and E. Sacco, "A multi-scale enriched model for the analysis of masonry panels," Int. J. Solids Struct., vol. 49, no. 6, pp. 865-880, Mar. 2012.

[14] D. Baraldi, E. Reccia, A. Cazzani, and A. Cecchi, "Comparative Analysis of Numerical Discrete and Finite Element Models: the Case of in-Plane Loaded Periodic Brickwork," Compos. Mech. Comput. Appl. An Int. J., vol. 4, no. 4, pp. 319-344, 2013.

[15] E. Reccia, A. Cazzani, and A. Cecchi, "FEM-DEM Modeling for Out-of-plane Loaded Masonry Panels: A Limit Analysis Approach," Open Civ. Eng. J., pp. 231-238, 2012.

[16] K. Sab, J. Dallot, and A. Cecchi, "Determination of the overall yield strength domain of outof-plane loaded brick masonry," Int. J. Multiscale Comput. Eng., 2007.

[17] Itasca Consulting Group, “3DEC 5.0.” Minneapolis, 2013.

[18] P. Cundall, "Formulation of a three-dimensional distinct element model-Part I. A scheme to detect and represent contacts in a system composed of many polyhedral blocks," Int. J. Rock Mech. Min. ..., vol. 25, no. 3, pp. 107-116, 1988.

[19] J. V. Lemos, "Discrete Element Modeling of Masonry Structures," Int. J. Archit. Herit., vol. 1, no. 2, pp. 190-213, May 2007. 